\title{
Existence and multiplicity of solutions for fractional Schödinger equation involving a critical nonlinearity
}

\author{
Yongzhen Yun ${ }^{1}$, Tianqing $A n^{1}$ and Guoju Y $\mathrm{e}^{1 *}$ (D)
}

\section{"Correspondence: ygihhu@163.com \\ ${ }^{1}$ College of Science, Hohai \\ University, Nanjing, China}

\begin{abstract}
In this paper, we investigate the fractional Schödinger equation involving a critical growth. By using the principle of concentration compactness and the variational method, we obtain some new existence results for the above equation, which improve the related results on this topic.
\end{abstract}

MSC: 35R11;35A15; 35B38

Keywords: Fractional Laplacian; Principle of concentration compactness; Fractional Schödinger equation; Weak solution; Variational method

\section{Introduction}

This paper is concerned with the following fractional Schödinger equation involving a critical nonlinearity:

$$
(-\Delta)^{\alpha} u(x)+V(x) u(x)=f(x, u(x))+\lambda|u(x)|^{2_{\alpha}^{*}-2} u(x), \quad x \in \mathbb{R}^{N},
$$

where $\alpha \in(0,1), 2 \alpha<N, 2_{\alpha}^{*}=\frac{2 N}{N-2 \alpha}, \lambda>0$ is a parameter, $V$ is potential and continuous function, while $f$ is a continuous function, both of them satisfying some conditions; $(-\Delta)^{\alpha}$ is the fractional Laplacian operator of order $\alpha$, which can be defined as $(-\Delta)^{\alpha} u=\mathscr{F}^{-1}\left(|\xi|^{2 \alpha} \mathscr{F} u\right)$, where $\mathscr{F}$ is the usual Fourier transform in $\mathbb{R}^{N}$.

Recently, fractional calculus attained more importance due to its wide applications in various field, such as diffusion, electrical circuits, control theory, blood flow phenomena, electro-analytical chemistry, etc.; for details, see [1-12] and the references therein. Problem (1.1) is related to the standing wave solutions of the following fractional nonlinear Schödinger equation of the form:

$$
i \frac{\partial \psi}{\partial t}(x, t)=(-\Delta)^{\alpha} \psi(x, t)+V(x) \psi(x, t)+g(x, t), \quad(x, t) \in \mathbb{R}^{N} \times \mathbb{R} .
$$

It was discovered by Laskin [13] when expanding the Feynman path integral, from Brownian-like to Lévy-like quantum mechanical paths. Recently, the fractional Schödinger equation has became a fundamental equation in fractional quantum mechanics, where, when $\varepsilon \rightarrow 0$ is taken in (1.2), the existence of solutions is very important; see [14] and

(c) The Author(s) 2019. This article is distributed under the terms of the Creative Commons Attribution 4.0 International License (http://creativecommons.org/licenses/by/4.0/), which permits unrestricted use, distribution, and reproduction in any medium, provided you give appropriate credit to the original author(s) and the source, provide a link to the Creative Commons license, and indicate if changes were made. 
the references therein. In the past few years, many works were devoted to establishing the existence and multiplicity of solutions of fractional Schödinger equation, see [15-22] and the references therein.

Recently, the existence of solutions of fractional Schödinger equation with perturbation was investigated, see [18-21]. Shang and Zhang [18] used the Ljusternik-Schnirelemann category theory to study the following fractional Schödinger equation with critical growth:

$$
\varepsilon^{2 \alpha}(-\Delta)^{\alpha} u(x)+V(x) u(x)=|u(x)|^{2_{\alpha}^{*}-2} u(x)+\lambda f(u(x)), \quad x \in \mathbb{R}^{N} .
$$

They proved that equation (1.3) has a nonnegative ground state solution in Nehari manifold. There are also some contributions considering the different forms of fractional Schödinger equation with critical growth, see [19-21]. It is worth mentioning that [19] studied the following fractional Schödinger equation:

$$
(-\Delta)^{\alpha} u(x)+V(x) u(x)=k(x) f(u(x))+\lambda|u(x)|^{2_{\alpha}^{*}-2} u(x), \quad x \in \mathbb{R}^{N} .
$$

By the principle of concentration compactness and the variational method, a nontrivial radially symmetric weak solution was obtained for (1.4).

It is well known that in the case of critical growth, the compactness of related embedding is lost, and then certain difficulties arise in the proving of the existence of solutions. In [18], Shang and Zhang obtained a nonnegative ground state solution for problem (1.3) in Nehari manifold by using the Ljusternik-Schnirelemann category theory. It seems that it is difficult to obtain the multiplicity result for problem (1.3). In [19], Zhang et al. used the fractional version of the principle of concentration compactness [23] and obtained new existence results for nontrivial radially symmetric weak solution of problem (1.4).

Motivated by these results, in this paper, we are interested in the fractional Schödinger equation involving critical exponent (1.1), which is more general than (1.4). By the Ekeland's variational principle [24] and the Mountain Pass Theorem [25], we obtain a new multiplicity result, which is different from the work of $[18,19]$. To the best of our knowledge, our work is the first attempt to use the principle of concentration compactness to study the existence and multiplicity of solutions for fractional Schödinger equation involving a critical nonlinearity as in (1.1).

To obtain our main result, we consider the potential function $V \in C\left(\mathbb{R}^{N}, \mathbb{R}\right)$ satisfying

$$
0<\inf _{x \in \mathbb{R}^{N}} V(x):=V_{0}<\lim _{|x| d \rightarrow \infty} V(x):=V(\infty)=+\infty
$$

The hypothesis $\left(V_{0}\right)$ was first introduced by Rabinowitz in [26]. Furthermore, we impose the following assumptions for the nonlinearity $f$ :

(f1) $f \in C\left(\mathbb{R}^{N} \times \mathbb{R}, \mathbb{R}\right), f(x, t)=o(|t|)$ as $t \rightarrow 0$ uniformly in $x \in \mathbb{R}^{N}$;

(f2) There exists a constant $C_{1}>0$ such that $|f(x, t)| \leq C_{1}\left(1+|t|^{2_{\alpha}^{*}-1}\right)$ for all $(x, t) \in \mathbb{R}^{N} \times \mathbb{R}$.

(f3) $F(x, t) \geq 0$ and $\frac{F(x, t)}{t^{2}} \rightarrow+\infty$ as $t \rightarrow \infty$ uniformly in $x \in \mathbb{R}^{N}$, where $F(x, t)=\int_{0}^{t} f(x, s) d s$

(f4) There exist $\theta>0$ such that $0<\theta F(x, t) \leq t f(x, t)$ for all $(x, t) \in \mathbb{R}^{N} \times \mathbb{R}$.

Now we state the main result. 
Theorem 1.1 Assume that $V$ and $f$ satisfy $\left(V_{0}\right)$ and (f1)-(f4). There exists $\lambda_{1}>0$ such that for any $0<\lambda<\lambda_{1}$, the problem (1.1) has three solutions.

This paper organized as follows. In Sect. 2, we give some preliminaries of fractional Sobolev spaces and prove some lemmas, which will be used later. Section 3 presents the proof of Theorem 1.1.

\section{Preliminaries}

Throughout this paper, $\|\cdot\|_{p}$ is the usual norm of $L^{p}\left(\mathbb{R}^{N}\right), B(x, r)$ is the open ball centered at $x$ with radius $r, C_{i}(i=1,2, \ldots)$ and $C$ denote positive constants, $\langle\cdot\rangle$ is the inner product at Hilbert space. We first provide a short review of fractional Sobolev spaces. A complete introduction can be found in $[14,27]$.

For $\alpha \in(0,1)$, the fractional Sobolev space $H^{\alpha}\left(\mathbb{R}^{N}\right)$ is defined by

$$
H^{\alpha}\left(\mathbb{R}^{N}\right):=\left\{u \in L^{2}\left(\mathbb{R}^{N}\right): \frac{|u(x)-u(y)|^{2}}{|x-y|^{N+2 \alpha}} \in L^{2}\left(\mathbb{R}^{N} \times \mathbb{R}^{N}\right)\right\}
$$

endowed with the natural norm

$$
\|u\|_{H^{\alpha}\left(\mathbb{R}^{N)}\right.}:=\left(\int_{\mathbb{R}^{N}} u^{2}(x) d x+\iint_{\mathbb{R}^{2 N}} \frac{|u(x)-u(y)|^{2}}{|x-y|^{N+2 \alpha}} d x d y\right)^{\frac{1}{2}} .
$$

Moreover, by Proposition 3.6 in [14], we have

$$
\iint_{\mathbb{R}^{2 N}} \frac{|u(x)-u(y)|^{2}}{|x-y|^{N+2 \alpha}} d x d y=\int_{\mathbb{R}^{N}}\left|(-\Delta)^{\frac{\alpha}{2}} u(x)\right|^{2} d x .
$$

Therefore, for any $v \in H^{\alpha}\left(\mathbb{R}^{N}\right)$, we have

$$
\iint_{\mathbb{R}^{2 N}} \frac{(u(x)-u(y))(v(x)-v(y))}{|x-y|^{N+2 \alpha}} d x d y=\int_{\mathbb{R}^{N}}(-\Delta)^{\frac{\alpha}{2}} u(x) \cdot(-\Delta)^{\frac{\alpha}{2}} v(x) d x .
$$

The working space $E$ is defined by

$$
E:=\left\{u \in H^{\alpha}\left(\mathbb{R}^{N}\right): \int_{\mathbb{R}^{N}} V(x) u^{2}(x) d x<+\infty\right\}
$$

Then $E$ is a Hilbert space with the norm

$$
\|u\|^{2}=\int_{\mathbb{R}^{N}}\left|(-\Delta)^{\frac{\alpha}{2}} u(x)\right|^{2} d x+\int_{\mathbb{R}^{N}} V(x) u^{2}(x) d x .
$$

We say that $u \in E$ is a weak solution of problem (1.1) if for any $v \in E$,

$$
\begin{gathered}
\int_{\mathbb{R}^{N}}(-\Delta)^{\frac{\alpha}{2}} u(x) \cdot(-\Delta)^{\frac{\alpha}{2}} v(x) d x+\int_{\mathbb{R}^{N}} V(x) u(x) v(x) d x \\
=\int_{\mathbb{R}^{N}}\left(f(x, u(x))+\lambda|u(x)|^{2_{\alpha}^{*}-2} u(x)\right) v(x) d x .
\end{gathered}
$$


The energy functional $I_{\lambda}: E \rightarrow \mathbb{R}$ associated with problem (1.1) is defined by

$$
\begin{aligned}
I_{\lambda}(u):= & \frac{1}{2} \int_{\mathbb{R}^{N}}\left|(-\Delta)^{\frac{\alpha}{2}} u(x)\right|^{2} d x+\int_{\mathbb{R}^{N}} V(x) u^{2}(x) d x \\
& -\int_{\mathbb{R}^{N}} F(x, u(x)) d x-\frac{\lambda}{2_{\alpha}^{*}} \int_{\mathbb{R}^{N}}|u(x)|^{2^{*}} d x .
\end{aligned}
$$

Under our assumptions, $I_{\lambda}$ is well defined in $E$ and $I_{\lambda} \in C^{1}(E, \mathbb{R})$. It is easy to obtain that $I_{\lambda}$ is Gâteaux-differentiable in $E$ and the critical points of $I_{\lambda}$ are solutions to problem (1.1).

Lemma 2.1 ([14]) Let $\alpha \in(0,1)$ and $N \geq 1$ be such that $2 \alpha<N$. Then there exists $C=$ $C(N, \alpha)>0$ such that

$$
\|u\|_{L^{2_{\alpha}^{*}\left(\mathbb{R}^{N}\right)}} \leq C\|u\|_{H^{\alpha}\left(\mathbb{R}^{N}\right)}
$$

for every $u \in H^{\alpha}\left(\mathbb{R}^{N}\right)$, where $2_{\alpha}^{*}=\frac{2 N}{N-2 \alpha}$ is the fractional critical exponent. Moreover, the embedding $H^{\alpha}\left(\mathbb{R}^{N}\right) \hookrightarrow L^{\gamma}\left(\mathbb{R}^{N}\right)$ is continuous for each $\gamma \in\left[2,2_{\alpha}^{*}\right]$ and is locally compact for $\gamma \in\left[2,2_{\alpha}^{*}\right)$.

Lemma $2.2([15,28])$ Assume that $\left(V_{0}\right)$ holds, then the embedding $E \hookrightarrow L^{p}\left(\mathbb{R}^{N}\right)$ is compact for all $2 \leq p<2_{\alpha}^{*}$.

Remark 2.3 From Lemmas 2.1 and 2.2, we know that the embedding $E \hookrightarrow L^{\gamma}\left(\mathbb{R}^{N}\right)$ is continuous for each $\gamma \in\left[2,2_{\alpha}^{*}\right]$, thus

$$
\|u\|_{L^{\gamma}\left(\mathbb{R}^{N)}\right.}^{\gamma} \leq C_{\gamma}\|u\|^{\gamma} \quad \text { for each } \gamma \in\left[2,2_{\alpha}^{*}\right]
$$

where $C_{\gamma}$ is the best constant for the embedding of $E$ into $L^{\gamma}\left(\mathbb{R}^{N}\right)$.

In the following lemma, we show that $I_{\lambda}$ satisfies the geometric structure of the Mountain Pass Theorem [25].

Lemma 2.4 The functional $I_{\lambda}$ satisfies the following condition:

(i) There exists $\lambda_{0}>0$ such that for any $\lambda \in\left(0, \lambda_{0}\right)$ and $\|u\|=\rho, I_{\lambda}(u) \geq \beta$ for some constants $\beta, \rho>0$.

(ii) There exists $e \in E$ satisfying $\|e\|>\rho$ such that $I_{\lambda}(e)<0$.

Proof (i) From conditions (f1) and (f2), for any $\varepsilon>0$, there exists a constant $C_{\varepsilon}$ depending on $\varepsilon$ such that

$$
|f(x, t)| \leq \varepsilon|t|+C_{\varepsilon}|t|^{2_{\alpha}^{*}-1}
$$

and

$$
|F(x, t)| \leq \frac{\varepsilon}{2}|t|^{2}+\frac{C_{\varepsilon}}{2_{\alpha}^{*}}|t|^{2 *}
$$


Therefore, for any $u \in E$, by (2.3) and (2.5), we have

$$
\begin{aligned}
I_{\lambda}(u) & =\frac{1}{2}\|u\|^{2}-\int_{\mathbb{R}^{N}} F(x, u(x)) d x-\frac{\lambda}{2_{\alpha}^{*}} \int_{\mathbb{R}^{N}}|u(x)|^{2_{\alpha}^{*}} d x \\
& \geq \frac{1}{2}\|u\|^{2}-\int_{\mathbb{R}^{N}}\left[\frac{\varepsilon}{2}|u(x)|^{2}+\frac{C_{\varepsilon}}{2_{\alpha}^{*}}|u(x)|^{2_{\alpha}^{*}}\right] d x-\frac{\lambda}{2_{\alpha}^{*}} \int_{\mathbb{R}^{N}}|u(x)|^{2_{\alpha}^{*}} d x \\
& =\frac{1}{2}\|u\|^{2}-\frac{\varepsilon}{2} \int_{\mathbb{R}^{N}}|u(x)|^{2} d x-\frac{C_{\varepsilon}}{2_{\alpha}^{*}} \int_{\mathbb{R}^{N}}|u(x)|^{2_{\alpha}^{*}} d x-\frac{\lambda}{2_{\alpha}^{*}} \int_{\mathbb{R}^{N}}|u(x)|^{2_{\alpha}^{*}} d x \\
& \geq \frac{1}{2}\|u\|^{2}-\frac{\varepsilon C_{2}}{2}\|u\|^{2}-\frac{C_{\varepsilon} C_{2_{\alpha}^{*}}}{2_{\alpha}^{*}}\|u\|^{2_{\alpha}^{*}}-\frac{\lambda C_{2_{\alpha}^{*}}}{2_{\alpha}^{*}}\|u\|^{2_{\alpha}^{*}} \\
& =\frac{1-\varepsilon C_{2}}{2}\|u\|^{2}-\frac{\left(C_{\varepsilon}+\lambda\right) C_{2_{\alpha}^{*}}}{2_{\alpha}^{*}}\|u\|^{2_{\alpha}^{*}} .
\end{aligned}
$$

Then there exists $\lambda_{0}>0$ such that for any $\lambda \in\left(0, \lambda_{0}\right)$, we can choose two positive constants $\beta, \rho>0$ such that $I_{\lambda}(u) \geq \beta$ for $\|u\|=\rho$.

(ii) For any $u \in E$, by condition (f3), we have

$$
\frac{I_{\lambda}(t u)}{t^{2}}=\frac{1}{2}\|u\|^{2}-\int_{\mathbb{R}^{N}} \frac{F(x, t u(x))}{t^{2} u^{2}(x)} u^{2}(x) d x-\frac{\lambda t^{2_{\alpha}^{*}}}{2_{\alpha}^{*}} \int_{\mathbb{R}^{N}}|u(x)|^{2_{\alpha}^{*}} d x,
$$

then

$$
\lim _{t \rightarrow+\infty} \frac{I_{\lambda}(t u)}{t^{2}} \leq \frac{1}{2}\|u\|^{2}-\lim _{t \rightarrow+\infty} \frac{\lambda t^{2_{\alpha}^{*}}}{2_{\alpha}^{*}} \int_{\mathbb{R}^{N}}|u(x)|^{2_{\alpha}^{*}} d x
$$

So, $I_{\lambda}(t u) \rightarrow-\infty$ as $t \rightarrow+\infty$, which means that there exists a large enough $t_{0}>0$ such that $I_{\lambda}\left(t_{0} u\right) \leq 0$. Therefore, we can choose $e:=t_{0} u \in E$ with $\|e\|>\rho$ such that $I_{\lambda}(e)<0$.

Lemma 2.5 Let $\lambda_{0}>0$ be given in Lemma 2.4. Then $\left\{u_{n}\right\}$ is a (PS) sequence of $I_{\lambda}$ which is bounded in $E$ for all $\lambda \in\left(0, \lambda_{0}\right)$.

Proof By condition (f4) and (2.3), for any $\left\{u_{n}\right\} \subset E$, we have

$$
\begin{aligned}
1+C+\left\|u_{n}\right\| \geq & I_{\lambda}\left(u_{n}\right)-\frac{1}{\theta}\left\langle I_{\lambda}^{\prime}\left(u_{n}\right), u_{n}\right\rangle \\
= & \left(\frac{1}{2}-\frac{1}{\theta}\right)\left\|u_{n}\right\|^{2}+\int_{\mathbb{R}^{N}}\left(u_{n}(x) f\left(x, u_{n}(x)\right)-\theta F\left(x, u_{n}(x)\right)\right) d x \\
& +\lambda\left(\frac{1}{\theta}-\frac{1}{2_{\alpha}^{*}}\right) \int_{\mathbb{R}^{N}}\left|u_{n}(x)\right|^{2_{\alpha}^{*}} d x \\
\geq & \left(\frac{1}{2}-\frac{1}{\theta}\right)\left\|u_{n}\right\|^{2}+\lambda\left(\frac{1}{\theta}-\frac{1}{2_{\alpha}^{*}}\right) C_{2_{\alpha}^{*}}\left\|u_{n}\right\|^{2_{\alpha}^{*}},
\end{aligned}
$$

which means that $\left\{u_{n}\right\}$ is bounded in $E$.

Let $\Omega_{c}\left(\mathbb{R}^{N}\right):=\left\{u \in C\left(\mathbb{R}^{N}\right): \operatorname{supp}(u)\right.$ is a compact subset of $\left.\mathbb{R}^{N}\right\}$ and denote by $\Omega_{0}\left(\mathbb{R}^{N}\right)$ the closure of $\Omega_{c}\left(\mathbb{R}^{N}\right)$ endowed with the norm $\|\eta\|_{\infty}=\sup _{x \in \mathbb{R}^{N}}|\eta(x)|$. For a measure $\mu$, 
we define

$$
\|\mu\|_{\Omega_{0}}:=\sup _{\Omega_{0}\left(\mathbb{R}^{N}\right),\|\eta\|_{\infty}=1}|(\mu, \eta)|,
$$

where $(\mu, \eta)=\int_{\mathbb{R}^{N}} \eta d \mu$. We assume that $J$ is a countable set.

Definition 2.6 ([16]) Let $\mathcal{M}\left(\mathbb{R}^{N}\right)$ denote the space of finite nonnegative Borel measures on $\mathbb{R}^{N}$. For any $\mu \in \mathcal{M}\left(\mathbb{R}^{N}\right)$, the equation $\mu\left(\mathbb{R}^{N}\right)=\|\mu\|_{\Omega_{0}}$ holds. We define that $\mu_{n} \rightarrow \mu$ weakly-* in $\mathcal{M}\left(\mathbb{R}^{N}\right)$ if $\left(\mu_{n}, \eta\right) \rightarrow(\mu, \eta)$ holds for all $\eta \in \bar{C}\left(\mathbb{R}^{N}\right)$ as $n \rightarrow \infty$.

Lemma 2.7 ([23]) Let $\left\{u_{n}\right\} \subset \dot{H}^{\alpha}\left(\mathbb{R}^{N}\right)$ be a sequence and $u_{n} \rightarrow u$ weakly as $n \rightarrow \infty$ and such that $\left|(-\Delta)^{\frac{\alpha}{2}} u_{n}\right|^{2} \rightarrow \mu$ and $\left|u_{n}\right|^{2_{\alpha}^{*}} \rightarrow v$ weakly-* in $\mathcal{M}\left(\mathbb{R}^{N}\right)$. Then, either $u_{n} \rightarrow u$ in $L_{\text {loc }}^{2_{\alpha}^{*}}\left(\mathbb{R}^{N}\right)$ or there exists a set of distinct points $\left\{x_{j}\right\}_{j \in J} \subset \bar{\Omega}$ (at most countable) and positive numbers $\left\{v_{j}\right\}_{j \in J}$ such that $v=|u|^{2_{\alpha}^{*}}+\sum_{j \in J} v_{j} \delta_{x_{j}}$, where $\Omega \subseteq \mathbb{R}^{N}$ is an open subset. If $\Omega$ is bounded, then there exist a positive measure $\tilde{\mu} \in \mathcal{M}\left(\mathbb{R}^{N}\right)$ with supp $\tilde{\mu} \subset \bar{\Omega}$ and positive numbers $\left\{\mu_{j}\right\}_{j \in J}$ such that $\mu=\left|(-\Delta)^{\frac{\alpha}{2}} u_{n}\right|^{2}+\tilde{\mu}+\sum_{j \in J} \mu_{j} \delta_{x_{j}}$.

Lemma 2.8 ([19]) Define

$$
\begin{aligned}
& \mu_{\infty}=\lim _{R \rightarrow \infty} \limsup _{n \rightarrow \infty} \int_{\left\{x \in \mathbb{R}^{N}:|x|>R\right\}}\left|(-\Delta)^{\frac{\alpha}{2}} u_{n}(x)\right|^{2} d x, \\
& \nu_{\infty}=\lim _{R \rightarrow \infty} \limsup _{n \rightarrow \infty} \int_{\left\{x \in \mathbb{R}^{N}:|x|>R\right\}}\left|u_{n}(x)\right|^{2^{*}} d x .
\end{aligned}
$$

Then the quantities $\mu_{\infty}$ and $\nu_{\infty}$ are well defined and satisfy

$$
\begin{aligned}
& \limsup _{n \rightarrow \infty} \int_{\mathbb{R}^{N}}\left|(-\Delta)^{\frac{\alpha}{2}} u_{n}(x)\right|^{2} d x=\int_{\mathbb{R}^{N}} d \mu+\mu_{\infty}, \\
& \limsup _{n \rightarrow \infty} \int_{\mathbb{R}^{N}}\left|u_{n}(x)\right|^{2_{\alpha}^{*}} d x=\int_{\mathbb{R}^{N}} d v+v_{\infty} .
\end{aligned}
$$

Lemma 2.9 ([19]) Let $\left\{u_{n}\right\} \subset \dot{H}^{\alpha}\left(\mathbb{R}^{N}\right)$ be such that $u_{n} \rightarrow u$ weakly-* in $\dot{H}^{\alpha}\left(\mathbb{R}^{N}\right)$, $\left|(-\Delta)^{\frac{\alpha}{2}} u_{n}\right|^{2} \rightarrow \mu$ and $\left|u_{n}\right|^{2_{\alpha}^{*}} \rightarrow v$ weakly-* in $\mathcal{M}\left(\mathbb{R}^{N}\right)$ as $n \rightarrow \infty$. Then $v_{i} \leq\left(S_{\alpha}^{-1} \mu\left\{x_{i}\right\}\right)^{\frac{2 *}{2}}$ for $i \in J$ and $v_{\infty} \leq\left(S_{\alpha}^{-1} \mu_{\infty}\right)^{\frac{2 *}{2}}$, where $x_{i}$, $v_{i}$ are from Lemma 2.7 and $\mu_{\infty}, v_{\infty}$ are given in Lemma 2.8, $S_{\alpha}$ is the best Sobolev constant of $\dot{H}^{\alpha}\left(\mathbb{R}^{N}\right) \hookrightarrow L^{2_{\alpha}^{*}}\left(\mathbb{R}^{N}\right)$, i.e.,

$$
S_{\alpha}=\inf _{u \in \dot{H}^{\alpha}\left(\mathbb{R}^{N}\right)} \frac{\int_{\mathbb{R}^{N}}\left|(-\Delta)^{\frac{\alpha}{2}} u(x)\right|^{2} d x}{\|u\|_{L^{2 *}\left(\mathbb{R}^{N}\right)}^{2}} .
$$

Now, we are ready to establish the following compactness result, which is used to prove our main result.

Lemma 2.10 There exists $\lambda_{*}>0$ such that for any $\lambda \in\left(0, \lambda_{*}\right)$, each bounded (PS) sequence for functional $I_{\lambda}$ contains a convergent subsequence.

Proof Let $\left\{u_{n}\right\} \subset E$ be a bounded (PS) sequence, i.e., there exists $C_{3}>0$ such that

$$
I_{\lambda}\left(u_{n}\right) \leq C_{3} \quad \text { and } \quad I_{\lambda}^{\prime}\left(u_{n}\right) \rightarrow 0 \quad \text { in } E \text {, as } n \rightarrow \infty .
$$


Passing to a subsequence, we still denote by $\left\{u_{n}\right\}$. Assume that $u_{n} \rightarrow u_{0}$ weakly in $E$. According to Lemma 2.2, we have $u_{n} \rightarrow u_{0}$ in $L^{p}\left(\mathbb{R}^{N}\right)$ and $u_{n} \rightarrow u_{0}$ a.e. in $\mathbb{R}^{N}$ as $n \rightarrow \infty$. Therefore, by Prokhorov's Theorem [29], there exist $\mu, v \in \mathcal{M}\left(\mathbb{R}^{N}\right)$ such that

$$
\left|(-\Delta)^{\frac{\alpha}{2}} u_{n}\right|^{2} \rightarrow \mu \quad \text { and } \quad\left|u_{n}\right|^{2_{\alpha}^{*}} \rightarrow v \quad \text { weakly- } * \text { in } \mathcal{M}\left(\mathbb{R}^{N}\right) \text { as } n \rightarrow \infty
$$

By Lemma 2.7, we have $u_{n} \rightarrow u_{0}$ in $L_{\text {loc }}^{2_{\alpha}^{*}}\left(\mathbb{R}^{N}\right)$ or $v=\left|u_{0}\right|^{2_{\alpha}^{*}}+\sum_{j \in J} v_{j} \delta_{x_{j}}$ as $n \rightarrow \infty$.

For any $\phi \in E$, we have

$$
\begin{aligned}
& \left\langle I_{\lambda}^{\prime}\left(u_{n}\right), \phi\right\rangle-\left\langle I_{\lambda}^{\prime}\left(u_{0}\right), \phi\right\rangle \\
& =\int_{\mathbb{R}^{N}}(-\Delta)^{\frac{\alpha}{2}}\left(u_{n}(x)-u_{0}(x)\right)(-\Delta)^{\frac{\alpha}{2}} \phi(x) d x+\int_{\mathbb{R}^{N}} V(x)\left(u_{n}(x)-u_{0}(x)\right) \phi(x) d x \\
& \quad-\int_{\mathbb{R}^{N}}\left(f\left(x, u_{n}(x)\right)-f\left(x, u_{0}(x)\right)\right) \phi(x) d x \\
& \quad-\lambda \int_{\mathbb{R}^{N}}\left(\left|u_{n}(x)\right|^{2_{\alpha}^{*}-2} u_{n}(x)-\left|u_{0}(x)\right|^{2_{\alpha}^{*}-2} u_{0}(x)\right) \phi(x) d x .
\end{aligned}
$$

Since $u_{n} \rightarrow u_{0}$ weakly in $E$,

$$
\begin{aligned}
& \int_{\mathbb{R}^{N}}(-\Delta)^{\frac{\alpha}{2}}\left(u_{n}(x)-u_{0}(x)\right)(-\Delta)^{\frac{\alpha}{2}} \phi(x) d x+\int_{\mathbb{R}^{N}} V(x)\left(u_{n}(x)-u_{0}(x)\right) \phi(x) d x \rightarrow 0 \\
& \quad \text { as } n \rightarrow \infty
\end{aligned}
$$

Since

$$
\left\{\left|u_{n}\right|^{2_{\alpha}^{*}-2} u_{n}-\left|u_{0}\right|^{2_{\alpha}^{*}-2} u_{0}\right\}_{n} \quad \text { is bounded in } L^{\frac{2_{\alpha}^{*}}{2_{\alpha}^{*}-1}}\left(\mathbb{R}^{N}\right)
$$

and

$$
\left|u_{n}\right|^{2_{\alpha}^{*}-2} u_{n}-\left|u_{0}\right|^{2_{\alpha}^{*}-2} u_{0} \rightarrow 0 \quad \text { a.e. in } \mathbb{R}^{N}
$$

then

$$
\left|u_{n}\right|^{2_{\alpha}^{*}-2} u_{n}-\left|u_{0}\right|^{2_{\alpha}^{*}-2} u_{0} \rightarrow 0 \quad \text { weakly in } L^{\frac{2_{\alpha}^{*}}{2_{\alpha}^{*}-1}}\left(\mathbb{R}^{N}\right)
$$

we obtain that

$$
\int_{\mathbb{R}^{N}}\left(\left|u_{n}(x)\right|^{2_{\alpha}^{*}-2} u_{n}(x)-\left|u_{0}(x)\right|^{2_{\alpha}^{*}-2} u_{0}(x)\right) \phi(x) d x \rightarrow 0 .
$$

In the following, we prove that

$$
\int_{\mathbb{R}^{N}}\left(f\left(x, u_{n}(x)\right)-f\left(x, u_{0}(x)\right)\right) \phi(x) d x \rightarrow 0 .
$$

As $\left\langle I_{\lambda}^{\prime}\left(u_{n}\right), \phi\right\rangle \rightarrow 0$, we get $\left\langle I_{\lambda}^{\prime}\left(u_{0}\right), \phi\right\rangle=0$, i.e., $I_{\lambda}^{\prime}\left(u_{0}\right)=0$. Thus,

$$
\int_{\mathbb{R}^{N}}\left|(-\Delta)^{\frac{\alpha}{2}} u_{0}(x)\right|^{2} d x+\int_{\mathbb{R}^{N}} V(x) u_{0}^{2}(x) d x
$$




$$
=\int_{\mathbb{R}^{N}} f\left(x, u_{0}(x)\right) u_{0}(x) d x+\frac{\lambda}{2_{\alpha}^{*}} \int_{\mathbb{R}^{N}}\left|u_{0}(x)\right|^{2_{\alpha}^{*}} d x .
$$

By (2.4) and Young inequality, we have

$$
\begin{aligned}
& \left|\left[f\left(x, u_{n}\right)-f\left(x, u_{0}\right)\right] \phi\right| \\
& \quad \leq\left|u_{n}\right||\phi|+C_{1}\left|u_{n}\right|^{2_{\alpha}^{*}-1}|\phi|+\left|u_{0}\right||\phi|+C_{2}\left|u_{0}\right|^{2_{\alpha}^{*}-1}|\phi| \\
& \quad \leq\left|u_{n}-u_{0}\right||\phi|+2\left|u_{0}\right||\phi|+C_{1}\left|u_{n}-u_{0}\right|^{2_{\alpha}^{*}-1}|\phi|+C_{2}\left|u_{n}-u_{0}\right|^{2_{\alpha}^{*}-1}|\phi| \\
& \quad \leq \varepsilon\left|u_{n}-u_{0}\right|^{2}+C_{\varepsilon}|\phi|^{2}+2\left|u_{0}\right||\phi|+\varepsilon\left|u_{n}-u_{0}\right|^{2_{\alpha}^{*}}+C_{1} C_{\varepsilon}|\phi|^{2_{\alpha}^{*}}+C_{2}\left|u_{0}\right|^{2_{\alpha}^{*}-1}|\phi| .
\end{aligned}
$$

Let

$$
\begin{aligned}
G_{\varepsilon, n}(x):= & \max \left\{\mid\left[f\left(x, u_{n}(x)\right)-f\left(x, u_{0}(x)\right)\right] \phi(x)\right]|-\varepsilon| u_{n}(x)-\left.u_{0}(x)\right|^{2} \\
& \left.-\varepsilon\left|u_{n}(x)-u_{0}(x)\right|^{2_{\alpha}^{*}}, 0\right\} .
\end{aligned}
$$

Then

$$
0 \leq G_{\varepsilon, n}(x) \leq C_{\varepsilon}|\phi|^{2}+2\left|u_{0}\right||\phi|+C_{1} C_{\varepsilon}|\phi|^{2_{\alpha}^{*}}+C_{2}\left|u_{0}\right|^{2_{\alpha}^{*}-1}|\phi| \in L^{1}\left(\mathbb{R}^{N}\right)
$$

and $G_{\varepsilon, n}(x) \rightarrow 0$ a.e. on $\mathbb{R}^{N}$. By the Lebesgue dominated convergence theorem, we have

$$
\int_{\mathbb{R}^{N}} G_{\varepsilon, n}(x) d x \rightarrow 0 \quad \text { as } n \rightarrow \infty .
$$

Therefore,

$$
\begin{aligned}
& \underset{n \rightarrow \infty}{\limsup }\left|\int_{\mathbb{R}^{N}}\left(f\left(x, u_{n}(x)\right)-f\left(x, u_{0}(x)\right)\right) \phi d x\right| \\
& \leq \limsup _{n \rightarrow \infty} \int_{\mathbb{R}^{N}} G_{\varepsilon, n}(x) d x+\varepsilon \limsup _{n \rightarrow \infty} \int_{\mathbb{R}^{N}}\left|u_{n}(x)-u_{0}(x)\right|^{2} d x \\
& \quad+\varepsilon \limsup _{n \rightarrow \infty} \int_{\mathbb{R}^{N}}\left|u_{n}(x)-u_{0}(x)\right|^{2_{\alpha}^{*}} d x \\
& \leq C_{3} \varepsilon .
\end{aligned}
$$

By the arbitrariness of $\varepsilon$, we have

$$
\int_{\mathbb{R}^{N}}\left(f\left(x, u_{n}(x)\right)-f\left(x, u_{0}(x)\right)\right) \phi(x) d x \rightarrow 0 .
$$

Next we will verify that $u_{n} \rightarrow u_{0}$ in $L^{2_{\alpha}^{*}}\left(\mathbb{R}^{N}\right)$. We claim that there exists a constant $\lambda_{*}>0$ such that $v_{i}=0$ and $v_{\infty}=0$ holds for any $0<\lambda<\lambda_{*}$ and $i \in J$. We argue by contradiction. Suppose that there exists $i_{0} \in J$ such that $v_{i_{0}}>0$ or $v_{\infty}>0$, then, by Lemma 2.9, we have

$$
\nu_{i_{0}} \leq\left(S_{\alpha}^{-1} \mu\left(x_{i_{0}}\right)\right)^{\frac{2_{\alpha}^{*}}{2}} .
$$


Let $\varphi \in C_{0}^{\infty}\left(\mathbb{R}^{N}\right)$ be such that $\varphi \in(0,1), \varphi=1$ in $B(0,1)$ and $\varphi=0$ in $\mathbb{R}^{N} \backslash B(0,2)$. For any $\varepsilon>0$, we define $\varphi_{\varepsilon}(x):=\varphi\left(\frac{x-x_{i_{0}}}{\varepsilon}\right)$, where $i_{0} \in J$. Using (2.1) and (2.6), one has

$$
\int_{\mathbb{R}^{N}}\left|u_{n}(x) \varphi_{\varepsilon}(x)\right|^{2_{\alpha}^{*}} d x \leq\left(S_{\alpha}^{-1} \iint_{\mathbb{R}^{2 N}} \frac{\left|u_{n}(x) \varphi_{\varepsilon}(x)-u_{n}(y) \varphi_{\varepsilon}(y)\right|^{2}}{|x-y|^{N+2 \alpha}} d x d y\right)^{\frac{2 \alpha}{2}}
$$

which means that

$$
\int_{\mathbb{R}^{N}}\left|u_{n}(x) \varphi_{\varepsilon}(x)\right|^{2_{\alpha}^{*}} d x \rightarrow \int_{\mathbb{R}^{N}} \varphi_{\varepsilon}^{2_{\alpha}^{*}} d v \quad \text { as } n \rightarrow \infty
$$

and

$$
\int_{\mathbb{R}^{N}} \varphi_{\varepsilon}^{2_{\alpha}^{*}} d v \rightarrow v\left(\left\{x_{i_{0}}\right\}\right)=v_{i_{0}}, \quad \text { as } \varepsilon \rightarrow 0 .
$$

By (f4), we obtain

$$
\begin{aligned}
\theta I_{\lambda}\left(u_{n}\right)-\left\langle I_{\lambda}^{\prime}\left(u_{n}\right), u_{n}\right\rangle \\
=\frac{\theta}{2}\left\|u_{n}\right\|^{2}-\theta \int_{\mathbb{R}^{N}} F\left(x, u_{n}(x)\right) d x-\frac{\lambda \theta}{2_{\alpha}^{*}} \int_{\mathbb{R}^{N}}\left|u_{n}(x)\right|^{2_{\alpha}^{*}} d x \\
\quad-\left\|u_{n}\right\|^{2}+\int_{\mathbb{R}^{N}} f\left(x, u_{n}(x)\right) u_{n}(x) d x+\lambda \int_{\mathbb{R}^{N}}\left|u_{n}(x)\right|^{2_{\alpha}^{*}} d x \\
=\left(\frac{\theta-2}{2}\right)\left\|u_{n}\right\|^{2}+\int_{\mathbb{R}^{N}}\left[f\left(x, u_{n}(x)\right) u_{n}(x)-\theta F\left(x, u_{n}(x)\right)\right] d x \\
\quad+\lambda\left(1-\frac{\theta}{2_{\alpha}^{*}}\right) \int_{\mathbb{R}^{N}}\left|u_{n}(x)\right|^{2_{\alpha}^{*}} d x \\
\geq \lambda\left(1-\frac{\theta}{2_{\alpha}^{*}}\right) \int_{\mathbb{R}^{N}}\left|u_{n}(x)\right|^{2_{\alpha}^{*}} d x \\
\geq \lambda\left(\frac{2_{\alpha}^{*}-\theta}{2_{\alpha}^{*}}\right) \int_{\mathbb{R}^{N}}\left|u_{n}(x) \varphi_{\varepsilon}(x)\right|^{2_{\alpha}^{*}} d x .
\end{aligned}
$$

Let $n \rightarrow \infty$, then we have $\theta C_{3} \geq \lambda\left(\frac{2_{\alpha}^{*}-\theta}{2_{\alpha}^{*}}\right) \int_{\mathbb{R}^{N}} \varphi_{\varepsilon}^{2_{\alpha}^{*}}(x) d x$. By (2.8) and (2.9), we know that

$$
2 C_{3} \geq \lambda\left(\frac{2_{\alpha}^{*}-\theta}{2_{\alpha}^{*}}\right) v_{i_{0}}
$$

Since $\left\{u_{n}\right\}$ is bounded in $E$, by the definition of $\varphi_{\varepsilon}$, we know that $\left\{u_{n} \varphi_{\varepsilon}\right\}$ is also bounded in $E$. Thus

$$
\left\langle I_{\lambda}^{\prime}\left(u_{n}\right), u_{n} \varphi_{\varepsilon}\right\rangle \rightarrow 0 \quad \text { as } n \rightarrow \infty
$$

which means that

$$
\begin{aligned}
& \int_{\mathbb{R}^{N}}(-\Delta)^{\frac{\alpha}{2}} u_{n}(x) \cdot(-\Delta)^{\frac{\alpha}{2}}\left(u_{n} \varphi_{\varepsilon}\right)(x) d x \\
& \quad=\int_{\mathbb{R}^{N}}\left[f\left(x, u_{n}(x)\right) u_{n}(x)+\lambda\left|u_{n}(x)\right|^{2_{\alpha}^{*}}-V(x)\left|u_{n}(x)\right|^{2}\right] \varphi_{\varepsilon}(x) d x+o(1) .
\end{aligned}
$$


By (2.4), we have

$$
t f(x, t) \leq \varepsilon|t|^{2}+C_{\varepsilon}|t|^{2_{\alpha}^{*}} .
$$

Then

$$
\begin{gathered}
\int_{\mathbb{R}^{N}}\left[f\left(x, u_{n}(x)\right) u_{n}(x)+\lambda\left|u_{n}(x)\right|^{2_{\alpha}^{*}}-V(x)\left|u_{n}(x)\right|^{2}\right] \varphi_{\varepsilon}(x) d x \\
\leq \int_{\mathbb{R}^{N}}\left[\varepsilon\left|u_{n}(x)\right|^{2}+C_{\varepsilon}\left|u_{n}(x)\right|^{2_{\alpha}^{*}}+\lambda\left|u_{n}(x)\right|^{2_{\alpha}^{*}}\right] \varphi_{\varepsilon}(x) d x .
\end{gathered}
$$

Since

$$
\int_{\mathbb{R}^{N}}\left|u_{n}(x)\right|^{2} \varphi_{\varepsilon}(x) d x=\int_{B\left(x_{i}, 2 \varepsilon\right)}\left|u_{n}(x)\right|^{2} \varphi_{\varepsilon}(x) d x \rightarrow \int_{B\left(x_{i}, 2 \varepsilon\right)}\left|u_{0}(x)\right|^{2} \varphi_{\varepsilon}(x) d x \quad \text { as } n \rightarrow \infty,
$$

and

$$
\int_{B\left(x_{i}, 2 \varepsilon\right)}\left|u_{0}(x)\right|^{2} \varphi_{\varepsilon}(x) d x \rightarrow 0 \quad \text { as } \varepsilon \rightarrow 0
$$

we get

$$
\begin{aligned}
& \underset{\varepsilon \rightarrow 0}{\limsup } \limsup _{n \rightarrow \infty} \int_{\mathbb{R}^{N}}\left[f\left(x, u_{n}(x)\right) u_{n}(x)+\lambda\left|u_{n}(x)\right|^{2_{\alpha}^{*}}-V(x)\left|u_{n}(x)\right|^{2}\right] \varphi_{\varepsilon}(x) d x \\
& \quad \leq\left(C_{\varepsilon}+\lambda\right) \limsup _{\varepsilon \rightarrow 0} \limsup _{n \rightarrow \infty} \int_{\mathbb{R}^{N}}\left|u_{n}(x)\right|^{2_{\alpha}^{*}} \varphi_{\varepsilon}(x) d x \\
& \quad \leq\left(C_{\varepsilon}+\lambda\right) \limsup _{\varepsilon \rightarrow 0} \int_{\mathbb{R}^{N}} \varphi_{\varepsilon}^{2_{\alpha}^{*}}(x) d x \\
& \quad=\left(C_{\varepsilon}+\lambda\right) v_{i_{0}} .
\end{aligned}
$$

In order to construct a contradiction with our claim, we choose $\lambda$ large enough satisfies $\lambda>C_{\varepsilon}$, then

$$
\begin{aligned}
& \underset{\varepsilon \rightarrow 0}{\limsup } \limsup _{n \rightarrow \infty} \int_{\mathbb{R}^{N}}\left[f\left(x, u_{n}(x)\right) u_{n}(x)+\lambda\left|u_{n}(x)\right|^{2_{\alpha}^{*}}-V(x)\left|u_{n}(x)\right|^{2}\right] \varphi_{\varepsilon}(x) d x \\
& \quad \leq 2 \lambda v_{i_{0}} .
\end{aligned}
$$

By (2.2), we have

$$
\begin{aligned}
\int_{\mathbb{R}^{N}} & (-\Delta)^{\frac{\alpha}{2}} u_{n}(x) \cdot(-\Delta)^{\frac{\alpha}{2}}\left(u_{n} \varphi_{\varepsilon}\right)(x) d x \\
= & \iint_{\mathbb{R}^{2 N}} \frac{\left(u_{n}(x)-u_{n}(y)\right)\left(u_{n}(x) \varphi_{\varepsilon}(x)-u_{n}(y) \varphi_{\varepsilon}(y)\right)}{|x-y|^{N+2 \alpha}} d x d y \\
= & \iint_{\mathbb{R}^{2 N}} \frac{\left(u_{n}(x)-u_{n}(y)\right)^{2} \varphi_{\varepsilon}(y)}{|x-y|^{N+2 \alpha}} d x d y \\
& +\iint_{\mathbb{R}^{2 N}} \frac{\left(u_{n}(x)-u_{n}(y)\right)\left(\varphi_{\varepsilon}(x)-\varphi_{\varepsilon}(y)\right) u_{n}(x)}{|x-y|^{N+2 \alpha}} d x d y .
\end{aligned}
$$


It is easy to get that

$$
\iint_{\mathbb{R}^{2 N}} \frac{\left(u_{n}(x)-u_{n}(y)\right)^{2} \varphi_{\varepsilon}(y)}{|x-y|^{N+2 \alpha}} d x d y \rightarrow \int_{\mathbb{R}^{N}} \varphi_{\varepsilon} d \mu \quad \text { as } n \rightarrow \infty
$$

and

$$
\int_{\mathbb{R}^{N}} \varphi_{\varepsilon} d \mu \rightarrow \mu\left(\left\{x_{i_{0}}\right\}\right) \quad \text { as } \varepsilon \rightarrow 0 .
$$

Using Hölder inequality, one has

$$
\begin{aligned}
& \iint_{\mathbb{R}^{2 N}} \frac{\left(u_{n}(x)-u_{n}(y)\right)\left(\varphi_{\varepsilon}(x)-\varphi_{\varepsilon}(y)\right) u_{n}(x)}{|x-y|^{N+2 \alpha}} d x d y \\
& \quad \leq \iint_{\mathbb{R}^{2 N}} \frac{\left|u_{n}(x)-u_{n}(y)\right|\left|\varphi_{\varepsilon}(x)-\varphi_{\varepsilon}(y)\right|\left|u_{n}(x)\right|}{|x-y|^{N+2 \alpha}} d x d y \\
& \quad \leq C\left(\iint_{\mathbb{R}^{2 N}} \frac{u_{n}^{2}(x)\left|\varphi_{\varepsilon}(x)-\varphi_{\varepsilon}(y)\right|^{2}}{|x-y|^{N+2 \alpha}} d x d y\right)^{\frac{1}{2}} .
\end{aligned}
$$

By the following equality (see (3.7) in [19])

$$
\limsup _{\varepsilon \rightarrow 0} \limsup _{n \rightarrow \infty} \iint_{\mathbb{R}^{2 N}} \frac{u_{n}^{2}(x)\left|\varphi_{\varepsilon}(x)-\varphi_{\varepsilon}(y)\right|^{2}}{|x-y|^{N+2 \alpha}} d x d y=0,
$$

as well as (2.11) and (2.12), we obtain that

$$
\mu\left(\left\{x_{i_{0}}\right\}\right) \leq 2 \lambda v_{i_{0}} \text { for any } i_{0} \in J .
$$

From (2.7) and (2.10), one has

$$
2 C_{3} \geq \lambda\left(\frac{2_{\alpha}^{*}-\theta}{2_{\alpha}^{*}}\right)\left(\frac{\lambda^{-1} S_{\alpha}}{2}\right)^{\frac{2_{\alpha}^{*}}{2 \alpha-2}}=\left(\frac{2_{\alpha}^{*}-\theta}{2_{\alpha}^{*}}\right)\left(\frac{S_{\alpha}}{2}\right)^{\frac{N}{2 \alpha}} \lambda^{\frac{2 \alpha-N}{2 \alpha}},
$$

which implies

$$
\lambda \geq\left(\frac{2_{\alpha}^{*}-\theta}{2 C_{3} 2_{\alpha}^{*}}\right)\left(\frac{S_{\alpha}}{2}\right)^{\frac{2 \alpha}{2}}:=\lambda_{*},
$$

in contradiction with our assumption $0<\lambda<\lambda_{*}$. Then, for any $i \in J, v_{i}=0$ and $v_{\infty}=0$ holds. Using Lemma 2.8, we have

$$
\limsup _{n \rightarrow \infty} \int_{\mathbb{R}^{N}}\left|u_{n}(x)\right|^{2_{\alpha}^{*}} d x=\int_{\mathbb{R}^{N}}\left|u_{0}(x)\right|^{2_{\alpha}^{*}} d x
$$

Since $\left|u_{n}-u_{0}\right|^{2_{\alpha}^{*}} \leq 2^{2_{\alpha}^{*}}\left(\left|u_{n}\right|^{2_{\alpha}^{*}}+\left|u_{0}\right|^{2 *}\right)$, by Fatou's Lemma, we obtain

$$
\begin{aligned}
& \int_{\mathbb{R}^{N}} 2^{2_{\alpha}^{*}+1}\left|u_{0}(x)\right|^{2_{\alpha}^{*}} d x \\
& \quad=\int_{\mathbb{R}^{N}} \liminf _{n \rightarrow \infty}\left(2^{2_{\alpha}^{*}}\left|u_{n}(x)\right|^{2_{\alpha}^{*}}+2^{2_{\alpha}^{*}}\left|u_{0}(x)\right|^{2_{\alpha}^{*}}-\left|u_{n}(x)-u_{0}(x)\right|^{2_{\alpha}^{*}}\right) d x
\end{aligned}
$$




$$
\begin{aligned}
& \leq \liminf _{n \rightarrow \infty} \int_{\mathbb{R}^{N}}\left(2^{2_{\alpha}^{*}}\left|u_{n}(x)\right|^{2_{\alpha}^{*}}+2^{2_{\alpha}^{*}}\left|u_{0}(x)\right|^{2_{\alpha}^{*}}-\left|u_{n}(x)-u_{0}(x)\right|^{2_{\alpha}^{*}}\right) d x \\
& \quad+2^{2_{\alpha}^{*}+1} \int_{\mathbb{R}^{N}}\left|u_{0}(x)\right|^{2_{\alpha}^{*}} d x-\underset{n \rightarrow \infty}{\limsup } \int_{\mathbb{R}^{N}}\left|u_{n}(x)-u_{0}(x)\right|^{2_{\alpha}^{*}} d x,
\end{aligned}
$$

which implies that

$$
\limsup _{n \rightarrow \infty} \int_{\mathbb{R}^{N}}\left|u_{n}(x)-u_{0}(x)\right|^{2_{\alpha}^{*}} d x=0
$$

then $u_{n} \rightarrow u_{0}$ in $L^{2_{\alpha}^{*}}\left(\mathbb{R}^{N}\right)$ as $n \rightarrow \infty$.

Note that $I_{\lambda}^{\prime}\left(u_{n}\right) \rightarrow 0$, and therefore

$$
\begin{aligned}
& \limsup _{n \rightarrow \infty} \int_{\mathbb{R}^{N}}\left|(-\Delta)^{\frac{\alpha}{2}} u_{n}(x)\right|^{2} d x \\
& \quad=\limsup _{n \rightarrow \infty}\left(\int_{\mathbb{R}^{N}} f\left(x, u_{n}(x)\right) u_{n}(x) d x+\lambda \int_{\mathbb{R}^{N}}\left|u_{n}(x)\right|^{2_{\alpha}^{*}} d x-\int_{\mathbb{R}^{N}} V(x) u_{n}^{2}(x) d x\right) \\
& \quad \leq \int_{\mathbb{R}^{N}} f\left(x, u_{0}(x)\right) u_{0}(x) d x+\lambda \int_{\mathbb{R}^{N}}\left|u_{0}(x)\right|^{2^{*}} d x-\int_{\mathbb{R}^{N}} V(x) u_{0}^{2}(x) d x \\
& \quad \leq \liminf _{n \rightarrow \infty} \int_{\mathbb{R}^{N}}\left|(-\Delta)^{\frac{\alpha}{2}} u_{n}(x)\right|^{2} d x,
\end{aligned}
$$

which means that

$$
\lim _{n \rightarrow \infty} \int_{\mathbb{R}^{N}}\left|(-\Delta)^{\frac{\alpha}{2}} u_{n}(x)\right|^{2} d x=\int_{\mathbb{R}^{N}}\left|(-\Delta)^{\frac{\alpha}{2}} u_{0}(x)\right|^{2} d x .
$$

Thus,

$$
\begin{aligned}
\limsup _{n \rightarrow \infty} & \int_{\mathbb{R}^{N}} V(x) u_{n}^{2}(x) d x \\
= & \limsup _{n \rightarrow \infty} \int_{\mathbb{R}^{N}}\left(\int_{\mathbb{R}^{N}} f\left(x, u_{n}(x)\right) u_{n}(x) d x+\lambda \int_{\mathbb{R}^{N}}\left|u_{n}(x)\right|^{2_{\alpha}^{*}} d x\right. \\
& \left.\quad-\int_{\mathbb{R}^{N}}\left|(-\Delta)^{\frac{\alpha}{2}} u_{n}(x)\right|^{2} d x\right) \\
= & \int_{\mathbb{R}^{N}} f\left(x, u_{0}(x)\right) u_{0}(x) d x+\lambda \int_{\mathbb{R}^{N}}\left|u_{0}(x)\right|^{2_{\alpha}^{*}} d x-\int_{\mathbb{R}^{N}}\left|(-\Delta)^{\frac{\alpha}{2}} u_{0}(x)\right|^{2} d x \\
= & \int_{\mathbb{R}^{N}} V(x) u_{0}^{2}(x) d x .
\end{aligned}
$$

Since $u_{n} \rightarrow u_{0}$ weakly in $E$, then by (2.13), we have

$$
\lim _{n \rightarrow \infty} \int_{\mathbb{R}^{N}}\left|(-\Delta)^{\frac{\alpha}{2}}\left(u_{n}-u_{0}\right)(x)\right|^{2} d x+\int_{\mathbb{R}^{N}} V(x)\left(u_{n}(x)-u_{0}(x)\right)^{2} d x=0,
$$

which implies that $u_{n} \rightarrow u_{0}$ in $E$.

Denote $B_{\rho}=\{u \in E:\|u\| \leq \rho\}$, where $\rho$ is given in Lemma 2.4. The following lemma expresses that the functional $I_{\lambda}$ has a local minimum in $\overline{B_{\rho}}$. 
Lemma 2.11 Assume that $\left(V_{0}\right)$ and (f4) hold. Let $\lambda_{0}>0$ be as in Lemma 2.4, then for every $\lambda \in\left(0, \lambda_{0}\right)$, there exists $u_{0} \in E$ such that

$$
I_{\lambda}\left(u_{0}\right)=\inf \left\{I_{\lambda}(u): u \in \overline{B_{\rho}}\right\}<0 .
$$

Proof Choose $\xi \in E$ such that $\int_{\mathbb{R}^{N}}|\xi(x)|^{2_{\alpha}^{*}} d x>0$. By (f4), for $t>0$ sufficiently large, we have

$$
\begin{aligned}
I_{\lambda}(t \xi) & =\frac{t^{2}}{2}\|\xi\|^{2}-\int_{\mathbb{R}^{N}} F(x, t \xi(x)) d x-\frac{\lambda t^{2_{\alpha}^{*}}}{2_{\alpha}^{*}} \int_{\mathbb{R}^{N}}|\xi(x)|^{2_{\alpha}^{*}} d x \\
& \leq \frac{t^{2}}{2}\|\xi\|^{2}-\frac{\lambda t^{2}}{2_{\alpha}^{*}} \int_{\mathbb{R}^{N}}|\xi(x)|^{2_{\alpha}^{*}} d x \\
& <0 .
\end{aligned}
$$

Therefore, letting $\rho>0$ be given as in Lemma 2.4, one has

$$
I_{\lambda}\left(u_{0}\right)=\inf \left\{I_{\lambda}(u): u \in \overline{B_{\rho}}\right\}<0 .
$$

\section{Proof of the main results}

In this section, we use the Ekeland's variational principle [24] and the Mountain Pass Theorem [25] to prove our main result.

Proof of Theorem 1.1 Due to (f1)-(f4), we easily know that 0 is a trivial solution of problem (1.1). Using the Ekeland's variational principle and Lemma 2.11, there exists a minimizing sequence $u_{n} \in \overline{B_{\rho}}$ such that $I_{\lambda}\left(u_{n}\right) \rightarrow \inf _{u \in \overline{B_{\rho}}} I_{\lambda}\left(u_{0}\right)$ and $I_{\lambda}^{\prime}\left(u_{n}\right) \rightarrow 0$ when $n \rightarrow \infty$. Therefore, by Lemma 2.10, we have that problem (1.1) has a nontrivial solution $u_{*}$ which satisfies $I_{\lambda}\left(u_{*}\right)<0$ and $\left\|u_{*}\right\| \leq \rho$.

Choose $\lambda_{1}:=\min \left\{\lambda_{0}, \lambda_{*}\right\}$, where $\lambda_{0}, \lambda_{*}$ are given in Lemmas 2.4 and 2.10. By the Mountain Pass Theorem and Lemma 2.4, there exists a (PS) sequence $\left\{u_{n}\right\} \subset E$ for $I_{\lambda}$ in $E$ for any $0<\lambda<\lambda_{1}$. According to Lemma 2.10, there exist a subsequence of $\left\{u_{n}\right\} \subset E$, still denoted by $\left\{u_{n}\right\}$, and $u_{* *} \in E$ such that $u_{n} \rightarrow u_{* *}$ in $E$ as $n \rightarrow \infty$. Moreover, $I_{\lambda}^{\prime}\left(u_{* *}\right)=0$ and $I_{\lambda}\left(u_{* *}\right) \geq \beta>0$. Hence, $u_{* *}$ is another nontrivial solution of problem (1.1).

From the above argument, we can conclude that problem (1.1) possesses three solutions such that $I_{\lambda}\left(u_{*}\right)<0=I_{\lambda}(0)<I_{\lambda}\left(u_{* *}\right)$ for all $0<\lambda<\lambda_{1}$. The proof is completed.

\section{Conclusion}

The fractional Schödinger equation is particularly important equation in fractional quantum mechanics. Recently, lots of papers have been published on the existence of solutions for fractional Schödinger equation. However, there are few of papers consider the existence of solutions for the fractional Schödinger equation with perturbation. In this paper, we study a new form of fractional Schödinger equation involving a critical nonlinearity (1.1). By using the principle of concentration compactness and the variational method, we obtain the existence and multiplicity of solutions for it; our work differs from earlier studies. Therefore, our results improve the corresponding results for this problem. In the future, we think that an accurate numerical solution for the fractional Schödinger equation can be obtained by using numerical methods, such as that developed in [30, 31]. 


\section{Acknowledgements}

The authors thank the anonymous referees for invaluable comments and insightful suggestions which improved the presentation of this manuscript.

\section{Funding}

This research is supported by the Fundamental Research Funds for the Central Universities (2019B64714, 2019B44914 2017B19714, 2017B07414), the Postgraduate Research \& Practice Innovation Program of Jiangsu Province (SJKY19_0431), Special Soft Science Research Projects of Technological Innovation in Hubei Province (2019ADC146), Natural Science Foundation of Jiangsu Province (BK20180500) and the National Key Research and Development Program of China (2018YFC1508100).

\section{Availability of data and materials}

Not applicable.

\section{Competing interests}

The authors declare that they have no competing interests.

\section{Authors' contributions}

Each of the authors contributed to each part of this study equally, all authors read and approved the final manuscript.

\section{Publisher's Note}

Springer Nature remains neutral with regard to jurisdictional claims in published maps and institutional affiliations.

Received: 13 September 2019 Accepted: 31 October 2019 Published online: 14 November 2019

\section{References}

1. Jajarmi, A., Arshad, S., Baleanu, D.: A new fractional modelling and control strategy for the outbreak of dengue fever. Phys. A, Stat. Mech. Appl. 535, 122524 (2019)

2. Baleanu, D., Jajarmi, A., Sajjadi, S.S., et al.: A new fractional model and optimal control of a tumor-immune surveillance with non-singular derivative operator. Chaos, Interdiscip. J. Nonlinear Sci. 29(8), 083127 (2019)

3. Jajarmi, A., Ghanbari, B., Baleanu, D.: A new and efficient numerical method for the fractional modelling and optimal control of diabetes and tuberculosis co-existence. Chaos, Interdiscip. J. Nonlinear Sci. 29(9), 093111 (2019)

4. Baleanu, D., Sajjadi, S.S., Jajarmi, A., et al.: New features of the fractional Euler-Lagrange equations for a physical system within non-singular derivative operator. Eur. Phys. J. Plus 134, 181 (2019)

5. Baleanu, D., Jajarmi, A., Asad, J.H.: Classical and fractional aspects of two coupled pendulums. Rom. Rep. Phys. 71(1), 103 (2019)

6. Veeresha, P., Prakasha, D.G., Qurashi, M.A., et al.: A reliable technique for fractional modified Boussinesq and approximate long wave equations. Adv. Differ. Equ. 2019, 253 (2019)

7. Tassaddiq, A., Khan, I., Nisar, K.S.: Heat transfer analysis in sodium alginate based nanofluid using MoS 2 nanoparticles: Atangana-Baleanu fractional model. Chaos Solitons Fractals 130, 109445 (2020)

8. Khan, O., Khan, N., Baleanu, D., et al.: Computable solution of fractional kinetic equations using Mathieu-type series. Adv. Differ. Equ. 2019, 234 (2019)

9. Nisar, K.S., Mondal, S.R. Belgacem, F.B.M.: On fractional kinetic equations $k$-Struve functions based solutions. Alex. Eng. J. 57(4), 3249-3254 (2018)

10. Shaikh, A.S., Nisar, K.S.: Transmission dynamics of fractional order typhoid fever model using Caputo-Fabrizio operator. Chaos Solitons Fractals 128, 355-365 (2019)

11. Shaikh, A., Tassaddiq, A., Nisar, K.S., et al.: Analysis of differential equations involving Caputo-Fabrizio fractional operator and its applications to reaction diffusion equations. Adv. Differ. Equ. 2019, 178 (2019)

12. Nisar, K.S., Gharsseldien, Z.M., Belgacem, F.B.M.: Solution of fractional distributed order reaction-diffusion systems with Sumudu transform. Nonlinear Stud. 24(4), 911-920 (2017)

13. Laskin, N.: Fractional quantum mechanics and Levy path integrals. Phys. Lett. A 268(4), 298-305 (2000)

14. Di Nezza, E., Palatucci, G., Valdinoci, E.: Hitchhike's guide to the fractional Sobolev spaces. Bull. Sci. Math. 136(5), 521-573 (2012)

15. Cheng, M.: Bound state for the fractional Schrödinger equation with unbounded potential. J. Math. Phys. 53, 043507 (2012)

16. Xiang, M., Zhang, B., Zhang, X.: A nonhomogeneous fractional $p$-Kirchhoff type problem involving critical exponent in $\mathbb{R}^{N}$. Adv. Nonlinear Stud. 17, 611-640 (2017)

17. Zhang, W., Tang, X., Zhang, J.: Infinitely many radial and non-radial solutions for a fractional Schrödinger equation. Comput. Math. Appl. 71, 737-747 (2016)

18. Shang, X., Zhang, J.: Ground states for fractional Schrödinger equations with critical growth. Nonlinearity 27, 187-207 (2014)

19. Zhang, X., Zhang, B., Repovš, D.: Existence and symmetry of solutions for critical fractional Schrödinger equations with bounded potentials. Nonlinear Anal. 142, 48-68 (2016)

20. Tao, F., Wu, X.: Exitence and multiplity of positive solution for fractional Schrödinger equations with critical growth. Nonlinear Anal., Real World Appl. 35, 158-174 (2017)

21. He, X., Zou, W.: Existence and concentration result for the fractional Schrödinger equations with critical nonlinearities. Calc. Var. 55, 91 (2016)

22. Yun, Y., An, T., Zuo, J., Zhao, D.: Infinitely many solutions for fractional Schrödinger equation with potential vanishing at infinity. Bound. Value Probl. 2019, 62 (2019)

23. Palatucci, G., Pisante, A.: Improved Sobolev embeddings, profile decomposition, and concentration compactness for fractional Sobolev spaces. Calc. Var. Partial Differ. Equ. 50, 799-829 (2014) 
24. Ekeland, I.: On the variational principle. J. Math. Anal. Appl. 47(2), 324-353 (1974)

25. Willem, M.: Minimax Theorems. Birkhäuser, Boston (1996)

26. Rabinowitz, P.H.: On a class of nonlinear Schrödinger equation. Z. Angew. Math. Phys. 43, 270-291 (1992)

27. Bisci, G.M., Radulescu, D., Servadei, R.: Variational Methods for Nonlocal Fractional Problems. Cambridge University Press, Cambridge (2016)

28. Torres, C.: Non-homogeneous fractional Schrödinger equation. arXiv:1311.0708

29. Bogachev, V.I.: Measure Theory, Vol.II. Springer, Berlin (2007)

30. Hajipour, M., Jajarmi, A., Baleanu, D.: On the accurate discretization of a highly nonlinear boundary value problem. Numer. Algorithms 79(3), 679-695 (2018)

31. Hajipour, M., Jajarmi, A., Malek, A., et al.: Positivity-preserving sixth-order implicit finite difference weighted essentially non-oscillatory scheme for the nonlinear heat equation. Appl. Math. Comput. 325, 146-158 (2018)

Submit your manuscript to a SpringerOpen ${ }^{\circ}$ journal and benefit from:

- Convenient online submission

- Rigorous peer review

- Open access: articles freely available online

- High visibility within the field

- Retaining the copyright to your article

Submit your next manuscript at $\boldsymbol{\nabla}$ springeropen.com 